Proceeding Series of the Brazilian Society of Computational and Applied Mathematics

\title{
Modelagem Matemática do Atrito Dinâmico na Haste Telescópica de um Equipamento Pneumático para Poda de Árvores
}

\author{
Antonio Carlos Valdiero ${ }^{1}$ \\ Rozimerli Raquel Milbeier Richter ${ }^{2}$ \\ Cristian Schoenmeier ${ }^{3}$ \\ Leonardo Bortolon Maraschin ${ }^{4}$ \\ Luiz Antonio Rasia ${ }^{5}$ \\ Departamento de Ciências Exatas e Engenharias, UNIJUÍ Câmpus Panambi, Panambi, RS
}

\begin{abstract}
Resumo. A presenta-se a modelagem matemática das principais características não lineares de atrito na haste telescópica do módulo de posicionamento de um equipamento pneumático para poda de árvores em aplicações de afastamento de vegetação próxima a linhas de energia elétrica. Os resultados ilustram as características do modelo matemático do atrito dinâmico.

Palavras-chave. Atrito não linear, Dinâmica do atrito, Atuador pneumático, Identificação de atrito, Matemática aplicada.
\end{abstract}

\section{Introdução}

Este trabalho apresenta a modelagem matemática das principais características não lineares de atrito na haste telescópica do módulo de posicionamento de um equipamento pneumático para poda de árvores em aplicações de manutenção de afastamento de vegetação próxima a linhas aéreas de transmissão de energia elétrica. $\mathrm{O}$ uso de atuadores pneumáticos é muito comum em aplicações industriais e agroflorestais [2,3,4], pois possui as seguintes vantagens: manutenção simples e fácil, baixo custo, boa relação força/tamanho e flexibilidade de instalação. Além disso, o ar comprimido é facilmente

\footnotetext{
1 valdiero@unijui.edu.br

2 rozimerlirichter@gmail.com

3 cristian.schoenmeier@gmail.com

4 leonardo.maraschin@unijui.edu.br

5 rasia@unijui.edu.br
} 
disponível na maioria das instalações industriais e em veículos tais como caminhões e tratores [2, 3]. Apesar destas vantagens, os atuadores pneumáticos apresentam dificuldades de modelagem devido a diversas características não lineares do sistema, tais como a zona morta na servoválvula [4], a compressibilidade do ar, a relação não linear da vazão nos orifícios de controle [2] e o atrito dinâmico [1, 4, 5].

Atualmente diversos trabalhos apresentam o estudo das características não lineares de atuadores pneumáticos [2, 3, 4]. Valdiero et al. [4] apresenta o estudo, o modelo matemático e uma proposta inovadora de identificação dos parâmetros para a zona morta em servovalvulas pneumáticas, onde o método utilizado para a compensação da zona morta é feito adicionando a inversa da função da zona morta no controle do sistema. O modelo matemático da válvula é dado como uma função que depende das tensões aplicadas à servo válvula por uma função das pressões, além de incluir uma modelagem completa do atrito dinâmico. Contudo este trabalho utiliza-se dos resultados das pesquisas antecedentes a fim de contribuir para a modelagem matemática e desenvolver um modelo matemático para o atrito dinâmico com a identificação dos parâmetros que contribua para o controle de posição de um cilindro pneumático linear para uma dada aplicação em um equipamento pneumático de poda para uma faixa de curso de trabalho em torno de 2,5 metros.

Com este propósito, a modelagem matemática desenvolvida no presente trabalho apresenta uma sistematização destas características não lineares de atrito, resultando em um modelo matemático não linear de $5^{\mathrm{a}}$ ordem para a haste de um atuador pneumático de grande curso utilizado para posicionamento do módulo de corte de um equipamento pneumático para poda de árvores em aplicações de manutenção de afastamento de vegetação próxima a cabos de transmissão de energia elétrica. As seções seguintes apresentam a descrição do sistema pneumático de posicionamento, a modelagem matemática da dinâmica do atrito, a identificação dos parâmetros de atrito por meio de testes experimentais ilustram as características do modelo de atrito desenvolvido e sua validação experimental.

\section{Descrição do Posicionador Pneumático}

O posicionador pneumático do equipamento de poda funciona com o ar comprimido que é fornecido à válvula a uma dada pressão de suprimento $\left(p_{s}\right)$ previamente regulada. Durante a operação, o sinal de controle $U_{T}$ energiza o solenóide da válvula de modo que uma força magnética resultante é aplicada no carretel da válvula, produzindo o deslocamento do carretel. O deslocamento do mesmo abre os orifícios de controle para que uma câmara do cilindro linear seja ligada a linha de pressão de suprimento e a outra seja ligada à pressão atmosférica $\left(p_{a t m}\right)$. Desta forma, produzindo uma diferença de pressão nas câmaras do cilindro linear, dando origem a uma força resultante movendo a massa $M$ (que inclui a massa da ferramenta de corte) presa à haste deste cilindro, em um deslocamento no sentido positivo ou negativo $y$, dependendo do sinal de entrada. Esta força gerada pelo atuador pneumático é proporcional às áreas transversais do êmbolo do cilindro e às pressões nas câmaras e é chamada de força pneumática. A Figura 1 mostra o desenho esquemático de um posicionador pneumático. Na seção seguinte apresenta-se a formulação do modelo matemático deste sistema dinâmico com enfoque no modelo do atrito dinâmico que ocorre nas vedações, além da identificação de seus parâmetros. 


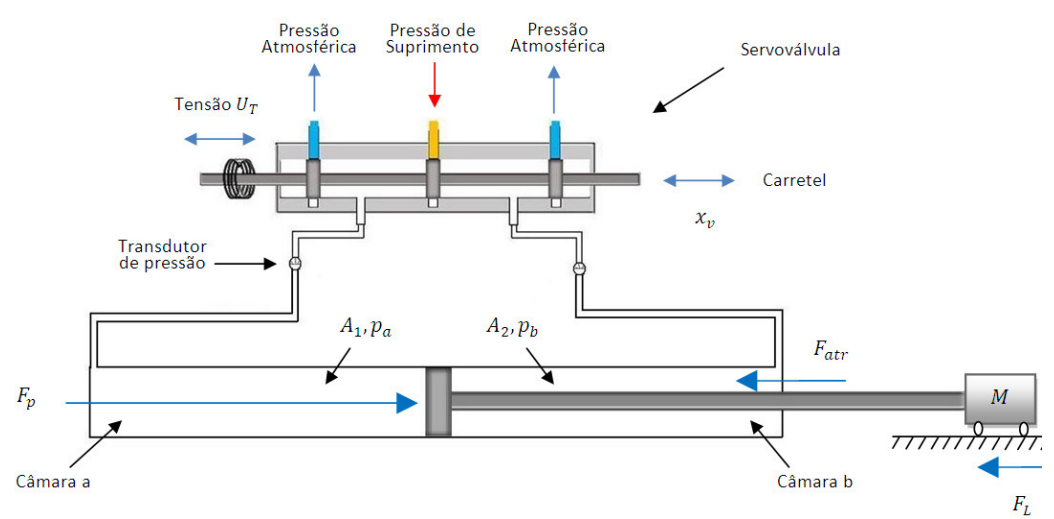

Figura 1: Desenho esquemático de um posicionador pneumático para equipamento de poda.

\section{Modelagem Matemática do Atrito Dinâmico Posicionador Pneumático}

Esta seção apresenta a modelagem matemática que descreve o comportamento dinâmico do posicionador pneumático e suas principais características não lineares. $\mathrm{O}$ modelo proposto é de $5^{\mathrm{a}}$ ordem e inclui as características não lineares do atrito [4]. A Figura 2 mostra o diagrama de blocos esquemático dos principais elementos incluídos na modelagem matemática utilizada para representar o comportamento dinâmico do posicionador pneumático, considerando-se a não linearidade da zona morta, a equação da vazão mássica, a dinâmica das pressões e a equação do movimento, que inclui a dinâmica do atrito.

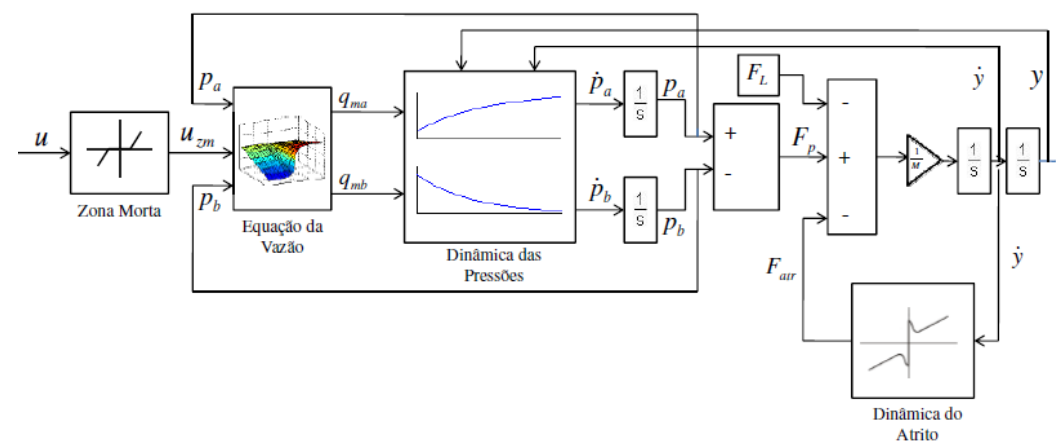

Figura 2: Diagrama esquemático da modelagem matemática do posicionador pneumático

A zona morta é uma imperfeição comum em válvulas pneumáticas, pois a largura do ressalto do carretel é maior que a largura do orifício. Dessa forma em alguns trechos do carretel não há passagem de ar. Esta não linearidade está localizada no sistema dinâmico como um bloco de entrada do sinal de controle, conforme mostrado na Figura 2, e é dada pela Eq. (1). 


$$
u_{z m}(t)=\left\{\begin{array}{l}
m d(u(t)-z m d) \quad \text { se } \quad u(t) \geq z m d \\
0 \quad \text { se } z m e<u(t)<z m d \\
m e(u(t)-z m e) \quad \text { se } u(t) \leq z m e
\end{array}\right.
$$

onde $u$ é o sinal de entrada, $u_{z m}$ é o valor de saída, zmd é o limite direito da zona morta, zme o valor esquerdo da zona morta, md é a inclinação direita da zona morta e me é a inclinação esquerda da zona morta.

A relação entre vazão-pressão nos orifícios da válvula é uma função não linear e depende da diferença de pressão no orifício da válvula e da abertura da válvula. Neste trabalho, será utilizado um inovador equacionamento da vazão mássica dadas pelas equações:

$$
\begin{gathered}
q_{m a}\left(u, p_{a}\right)=g_{1}\left(p_{a}, \operatorname{sign}(u)\right) \operatorname{arctg}(2 u) \\
q_{m b}\left(u, p_{b}\right)=g_{2}\left(p_{b}, \operatorname{sign}(u)\right) \operatorname{arctg}(2 u)
\end{gathered}
$$

onde $g_{1}$ e $g_{2}$ são funções sinal dadas por:

$$
\begin{aligned}
& g_{1}\left(p_{a}, \operatorname{sign}(u)\right)=\beta \Delta p_{a}=\left\{\begin{array}{lll}
\left(p_{\text {sup }}-p_{a}\right) \beta^{\text {ench }} & \text { se } & u \geq 0 \\
\left(p_{a}-p_{\text {atm }}\right) \beta^{\text {esv }} & \text { se } & u<0
\end{array}\right. \\
& g_{2}\left(p_{b}, \operatorname{sign}(u)\right)=\beta \Delta p_{b}=\left\{\begin{array}{lll}
\left(p_{\text {sup }}-p_{b}\right) \beta^{\text {ench }} & \text { se } & u<0 \\
\left(p_{b}-p_{\text {atm }}\right) \beta^{\text {esv }} & \text { se } & u \geq 0
\end{array}\right.
\end{aligned}
$$

onde $p_{\text {sup }}$ é a pressão de suprimento, $p_{\text {atm }}$ é a pressão atmosférica e $\beta^{\text {ench }}$ e $\beta^{\text {esv }}$ são coeficientes constantes característicos respectivamente do enchimento e do esvaziamento das câmaras do cilindro.

O modelo para a dinâmica das pressões é obtido a partir da equação da continuidade e resulta em duas equações não lineares de primeira ordem:

$$
\begin{gathered}
\dot{p}_{a}=-\frac{A \gamma \dot{y}}{A y+V_{a 0}} p_{a}+\frac{R \gamma T}{A y+V_{a 0}} q_{m a}\left(p_{a}, u\right) \\
\dot{p}_{b}=\frac{A \gamma \dot{y}}{V_{b 0}-A y} p_{b}-\frac{R \gamma T}{V_{b 0}-A y} q_{m b}\left(p_{b}, u\right)
\end{gathered}
$$

onde $T$ é a temperatura do ar de suprimento, $R$ é a constante universal dos gases, $q_{m a}=d m_{a} / d t$ é a vazão mássica na câmara $a$ do cilindro, $p_{a}$ é a pressão na câmara $a$ do cilindro, $\gamma=C_{p} / C_{v}$ é uma relação entre os calores específicos do ar com $C_{p}$ sendo o calor específico do ar à pressão constante e $C_{v}$ o calor especifico do ar a volume constante, $A$ é a área do êmbolo, $y$ é o deslocamento do êmbolo e $V_{a 0}$ é o volume da câmara $a$ na posição inicial $y=0$, incluindo o volume da tubulação. Assume-se que as vazões mássicas são funções não lineares das pressões nas câmaras dos cilindros e da tensão $u$ aplicadas a servoválvula, ou seja, $q_{m a}=q_{m a}\left(p_{a}, u\right)$ e $q_{m b}=q_{m b}\left(p_{b}, u\right)$.

A aplicação da $2^{\text {a }}$ Lei de Newton para o equilíbrio das forças no êmbolo do atuador pneumático resulta em:

$$
M \ddot{y}+F_{a t r}=F_{p}
$$


onde $M$ é a massa deslocada, $\ddot{y}$ é a aceleração do cilindro, $F_{p}$ é a força pneumática, dada pela diferença de pressão nas câmaras do cilindro, ou seja, $A\left(p_{a}-p_{b}\right)$ e $F_{a t r}$ é a força de atrito dinâmico adotada na forma do chamado modelo LuGre [1] adaptado conforme Valdiero [5] na seguinte equação:

$$
F_{a t r}=\sigma_{0} z+\sigma_{1} \dot{z}+c_{d} \dot{y}^{2}
$$

onde $\sigma_{0}$ representa o coeficiente de rigidez das deformações microscópicas, $z$ é um estado interno não mensurável que representa a deformação média que ocorre entre as superfícies, $\sigma_{1}$ é um coeficiente de amortecimento associado a taxa de variação de $z, c_{d}$ é o coeficiente de arraste e $\dot{y}$ é a velocidade relativa entre as superfícies.

A dinâmica da microdeformação z é dada por:

$$
\frac{d z}{d t}=\dot{y}-\alpha(z, \dot{y}) \frac{\sigma_{0}}{g_{s s}(\dot{y})}|\dot{y}| z
$$

onde $g_{s s}(\dot{y})$ é uma função positiva que descreve parte das características do atrito em regime permanente, e é descrita por:

$$
g_{s s}(\dot{y})=F_{c}+\left(F_{s}-F_{c}\right) e^{-\left(\frac{\dot{y}}{\dot{y} s}\right)^{2}}
$$

onde $F_{c}$ é o atrito de Coulomb, $F_{s}$ é o atrito estático e $\dot{y}_{s}$ é a velocidade de Stribeck. A função $\alpha(z, \dot{y})$ é incorporada ao modelo LuGre, conforme propõem Valdiero et al. [4] e é usada para representar o regime de atrito estático em velocidades baixíssimas.

Os parâmetros do modelo matemático são identificados na bancada experimental descrita a seguir.

\section{Descrição da bancada e Resultados de Testes Experimentais de Identificação do Atrito}

A bancada experimental de testes utilizada é composta por um microcomputador interligado uma placa eletrônica dSPACE 1104 responsável pela captura e aquisição dos sinais da bancada de testes, a qual utiliza a integração dos softwares MatLab/Simulink e ControlDesk, permitindo a captura e o controle em tempo real. As características técnicas dos principais componentes utilizados na bancada experimental estão descritas em [3]. A identificação dos parâmetros estáticos de atrito provêm de diversos experimentos em malha aberta, tendo como entrada o sinal de controle em tensão, variando de velocidades baixas até a máxima velocidade de trabalho do sistema. Os testes experimentais foram realizados em com o cilindro pneumático do equipamento de poda disponível NIMeP/UNIJUÍ. A força de atrito em regime permanente pode ser representada em uma curva experimental que é chamada de mapa estático, onde os parâmetros estáticos do atrito podem ser facilmente identificados. No procedimento de ajuste dos parâmetros de atrito é utilizado o algoritmo nlinfit do software MatLab, conforme mostrado na Figura 3. A partir deste algoritmo e de simulações computacionais é possível obter o melhor ajuste para os parâmetros estáticos 
dinâmicos de atrito, conforme metodologia descrita por Valdiero [5].

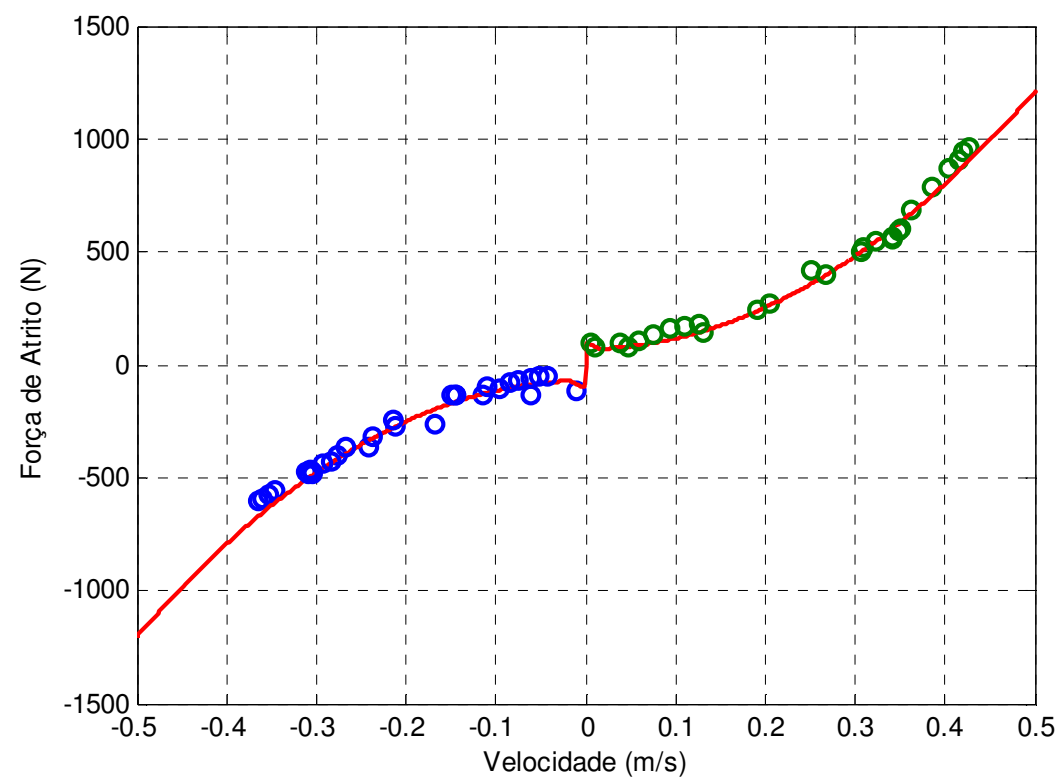

Figura 3: Determinação do mapa estático do atrito em um cilindro especial de dupla ação e haste simples utilizado no equipamento de poda.

A Tabela 1 apresenta os resultados obtidos para os valores dos parâmetros do modelo não linear de atrito utilizados na modelagem matemática.

Tabela 1: Valores dos parâmetros do modelo dinâmico não linear do atrito no posicionador pneumático utilizado no equipamento para poda de árvores.

\begin{tabular}{|c|c|c|}
\hline Descrição do Parâmetro & Simbologia & Valor \\
\hline Atrito estático & $F_{s}$ & $100 \mathrm{~N}$ \\
\hline Atrito Coulomb & $F_{c}$ & $70 \mathrm{~N}$ \\
\hline Coeficiente de arraste & $c_{d}$ & $4546,7 \mathrm{~N} . \mathrm{s}^{2} / \mathrm{m}^{2}$ \\
\hline Velocidade Stribeck & $\dot{y}_{s}$ & $0,01 \mathrm{~m} / \mathrm{s}$ \\
\hline Coeficiente de rigidez das microdeformações & $\sigma_{0}$ & $1,4 \times 10^{6} \mathrm{~N} / \mathrm{m}$ \\
\hline Coeficiente de amortecimento das microdeformações & $\sigma_{1}$ & $50 \mathrm{~N} . \mathrm{s} / \mathrm{m}$ \\
\hline
\end{tabular}

Os resultados obtidos na modelagem matemática do atrito e na identificação dos seus parâmetros foram utilizados para simulação do comportamento dinâmico da haste telescópica do equipamento para poda de árvores [3]. 


\section{Conclusões}

Os resultados obtidos com a modelagem matemática do atrito dinâmico no atuador pneumático utilizado na haste telescópica do equipamento para poda de árvores permite prever o comportamento e os aspectos de seu funcionamento. $\mathrm{O}$ conhecimento das características não lineares do atrito que dominam a dinâmica do movimento da haste é importante para melhoria da qualidade e do desempenho nas tarefas de posicionamento da ferramenta de corte de galhos. Estratégias de controle baseadas no modelo matemático permitem a compensação das características de atrito indesejadas, linearizando o sistema e diminuindo os efeitos de degradação tais como a perda de movimento e o fenômeno de adere-desliza [5]. Os resultados serão utilizados num esquema de compensação de atrito do controlador de posição da haste telescópica do equipamento para poda de árvores.

\section{Agradecimentos}

$\mathrm{O}$ presente trabalho foi realizado com apoio do $\mathrm{CNPq}$, Conselho Nacional de Desenvolvimento Científico e Tecnológico - Brasil. Os autores são agradecidos à CELPE (Companhia Energética de Pernambuco) pelo apoio financeiro no âmbito Programa P\&D da ANEEL (código ANEEL PD-0043-0311/2011).

\section{Referências}

[1] C. Canudas-de-Wit and P. Lischinsky, Adaptive friction compensation with partially known dynamic friction model, International Journal of Adaptive Control and Signal Processing, vol.11, 65-80, (1997).

[2] Z. Rao and G. M. Bone, Nonlinear Modeling and Control of Servo Pneumatic Actuators, IEEE Transactions on Control Systems Technology, vol. 16, (2008).

[3] R. R. M. Richter, Modelagem matemática e controle de posição de um atuador linear acionado pneumaticamente, Dissertação de Mestrado em Modelagem Matemática, UNIJUí, (2013).

[4] A. C. Valdiero, C. S. Ritter, C. F. Rios, M. Rafikov, "NonLinear Mathematical Modeling in Pneumatic Servo Position Applications", Mathematical Problems in Engineering (Online), vol. 2011, pp.1 - 16, (2011).

[5] A. C. Valdiero, Modelagem Matemática de Robôs Hidráulicos, Ijuí, UNIJUÍ, (2012). 